\title{
Research on Dynamic Management System of Double - Deep Rack for Automated Warehouse
}

\author{
Ma Yunfeng, Gao Yang* \\ School of Management \\ Wuhan University of Science and Technology \\ Qingshan district of Wuhan \\ Hubei, 430081 \\ E-mail:729500338@qq.com
}

\begin{abstract}
In order to reduce the land cost of the warehouse, improve the competitiveness of enterprises. One of the approaches increasing space utilization is to reduce passageway as far as possible. Compared with single-double rack, doubledeep rack can save half passageway space, hence can realize more compact warehousing. Firstly, an interleaving time model, for which both the run time of automated crane and the handling time of stacking crane are taken into consideration; secondly, a dynamic storage optimization method is put forward, which, in relation to random storage method, can shorten interaction time, and reduce reshuffle as far as possible; at last, simulation experiment is carried out, which indicates that the optimized storage method can improve operation efficiency.
\end{abstract}

Keywords-Automated warehouse; double-deep rack; dual command; interaction time; dynamic optimization

\section{INTRODUCTION}

Presently, warehousing system is developing in the directions of compactification of storage space and promptness of service time. Compared with traditional warehousing system, compact warehousing system differs mainly in two aspects: compactification of storage space and promptness of service time. In compact warehousing system, the number of passageways is reduced to increase space utilization, and the space for passageways in traditional warehousing system also is transformed into storage space, so that the working space is greatly reduced. On the other hand, compact warehousing system realizes coordination and optimization of operation process and operation time in the links of storing and retrieving, sorting, and dispatching, to address customers' diversified and individualized demands.

At present, the literatures on research of compact warehouse mainly focus on two aspects. One aspect is storage layout design. Iris F A [1] focused on dynamic design method of control strategy, and determined the placement rule of interleaving facilities, to reduce response time. Yang Hagen [2] designed a power-driven intensive mobile rack system, which helps reduce storage space and raising the efficiency of warehousing and ex-warehousing of cargos. Hwan Seong Kim [3] replaced traditional rack with mobile rack to raise the storage space efficiency of warehouse, and put forward a sequential optimization heuristic algorithm based on single operation sequence. Concerning storage layout, there are few people applying double-deep rack in automated warehousing system. The other aspect is optimization of storage method. Warren H. Hausman [4] put forward a storage optimization method based on comparison of single operation time of random storage, turnover rate storage and class-based storage. Seval Ene. Nursel Ozturk [5] considered batch processing and routine problem simultaneously in research of warehousing operation with a purpose of realizing minimization of transportation cost. Russell D. Meller [6] built an analysis model to estimate the handling capacity of double-shuttle and three- shuttle systems, and demonstrated the model in use with existing three-spindle system model, which indicates that the advantage of the system is reduction in waiting time. Deng Aimin [7] built a time-based multi-objective goods allocation position optimization model, for which the main factors taken into account include effective recording related data before and after shifting of cargo in automated stereoscopic warehouse, high efficiency of warehousing and exwarehousing of cargo, and shortest displacement distance of cargo. Through optimization, the management cost of warehouse is reduced, and the operation efficiency is raised. $\mathrm{Gu}$ Jinxiang [8] summarized the problems of warehouse operation plan, and explained that planning model and method content can be used in warehouse operation. Kees Jan Roodbergen [9], in review of automated warehousing system literatures, pointed out that most scholars' researches focused on stacking crane executing single command, and few scholars' researches focused on double-command. Shen Guwen [10], in research of compact warehousing system performance and action strategy, proposed to raise the operation efficiency of system via double- shuttle stacking crane, and built an expectation cycle time model of singleinstruction and double-instruction.

Presently, there are few Chinese literatures on research of dynamic goods allocation position of automated warehousing system, and combination of double-deep rack with dualcommand. This paper is intended to focus on application of double-deep rack in automated warehousing system, and execution of automated crane of dual-command to shorten interaction time, so as to shorten the total time. 


\section{PROBLEM DESCRIPTION AND MODEL}

\section{A. Problem Description}

Working principle of system: firstly, goods are dispatched into tray (it is possible that more than one article are dispatched into one tray); secondly, the tray reaches the place for storage of rack under the instruction from computer, and the location is recorded in computer system; at last, the tray is placed in goods place with automated crane. Upon receiving an instruction of searching goods, computer will get the position of the tray via information storage, and directly order the automated crane to search the tray. After sorting, the tray returns to the feed place of warehouse for reuse after being cleared up.

Double-deep rack refers to placing two rows of racks together. In this way, each goods allocation position is for two units of articles, but reshuffle is needed. For purpose of this paper, the goods allocation position near the outside of aisle is taken as the first goods allocation position; and that near the inside as the second goods allocation position.

For the problem of reshuffle arising from the application of double-deep rack in automated warehousing system, it is proposed to replace traditional single-shuttle stacking crane with double-shuttle stacking crane. With double-shuttle stacking crane, reshuffle can be done without backwards and forwards transportation of goods with the aid of a buffer zone, having raised the operation efficiency of automated warehousing system.

Dual command means that stacking crane needs to do two actions at a time, namely storage and retrieving. In the process of executing dual command, interaction time exists. Interaction time means the time to be taken to search goods after finishing the action of storage. Single command means doing one action at a time only, storage or retrieving.

\section{B. $\quad$ Model Building}

In this paper, it is planned to apply double-deep rack in automated warehousing system, and realize automated crane executing dual command. To this end, the following hypotheses are put forward:

(1) Stacking crane can store or retrieve unit articles at a time; (2)The time taken by stacking crane to handle cargo at I/O point is ignored; (3)All goods allocation positions are the same in size; (4)Stacking crane serves racks on both sides of which there are aisles and performs transportation at I/O point; (5)The horizontal velocity and vertical velocity of stacking crane are equal: $\mathrm{VH}=\mathrm{VV}=1 ;(6)$ The time taken by stacking crane to store goods and that to retrieve goods are equal.

The parameters of the model are as follows:

Si refers to set of goods allocation positions where $\mathrm{i}$ goods are stored; $\mathrm{Rm}$ refers to set of goods allocation positions for searching $\mathrm{m}$ goods; $\mathrm{K}$ refers to the quantity of automated crane; T IS refers to the time taken to travel from I/O point to goods allocation position S; TSR refers to the time taken to goods allocation position $S$ to goods allocation position $\mathrm{R}$;TRO refers to returning from goods allocation position $\mathrm{R}$ to
I/O point; $\Psi$ refers to the time taken by automated crane to handle goods at goods allocation position.

Decision variable:

$$
y_{i m}^{S R}=\left\{\begin{array}{l}
1, \text { automated crane executes dual command of storing } \\
\mathrm{i} \text { goods and } \mathrm{m} \text { goods, and stores } \mathrm{i} \text { in } \mathrm{S}, \text { and } \mathrm{m} \text { in } \mathrm{R} \\
0, \text { otherwise }
\end{array}\right.
$$

If the command is disequilibrium, and multiple automated dollies execute dual command, some automated dollies will become idle, while others will be always busy. In view of this, to put automated crane into rational utilization, a dual command time model for one crane travelling in multiple aisles is built, as below:

$$
\min T_{2}=\sum_{i \in S_{i}} \sum_{m \in R_{m}} y_{i m}^{S R}\left(T_{I S}+T_{S R}+T_{R O}+\psi\right)
$$

Formula (2) is for calculating the sum of travelling time of one automated crane executing dual command in multiple aisles (from I/O point to storage position, to search position, and to I/O point) and handling time in cargo place thereof.

The constraint conditions are:

$$
\begin{gathered}
\sum_{i \in S_{i}} \sum_{m \in R_{m}} y_{i m}^{S R}=1 \quad i=1,2 \ldots I ; m=1,2 \ldots M \\
\sum_{i \in S_{i}} y_{i m}^{S R} \leq 1 \quad m=1,2 \ldots M \\
\sum_{i \in S_{i}} y_{i m}^{S R} \leq 1 \quad m=1,2 \ldots M \\
y_{i m}^{S R} \in\{0,1\}
\end{gathered}
$$

Formula (3) means only one goods allocation position can be occupied for each time of storing or retrieving; Formula (4) means at most one operation of storing is included in a dual command action; Formula (5) means at most one operation of retrieving is included in a dual command action; Formula (6) indicates that $y_{i m}^{S R}$ is a variable of which the value is 0 or 1 .

\section{ALGORITHM DESIGN}

The time for sorting the first goods allocation position is longer than that for sorting the second goods allocation position; when stacking crane stores or retrieves goods into or from the second goods allocation position, and the first goods allocation position is not empty, it needs to move the cargo in the first goods allocation position to an automated crane for temporary storage, then sort the cargo in the second goods allocation position, and lastly put the cargo from the first goods allocation position back into the first goods allocation position again.

$\mathrm{I} / \mathrm{O}$ point is taken as the origin of coordinates $(0,0), \mathrm{X}$-axis refers to horizontal travelling of stacking crane, $\mathrm{Y}$-axis refers to vertical travelling of stacking crane, and the travelling velocity of automated crane is unit time per second; the time taken by automated crane to retrieve cargo from the outside rack is $\mathrm{t} 1$ second, that to retrieve cargo from the inside rack is 
$\mathrm{t} 2$ second, and $\mathrm{t} 2=2 \mathrm{t} 1$; the correlation between aisle quantity (A) and rack quantity ( $R$ ) is: $R=2 A$, provided that two rows of racks are parallel. Fig. 1 shows the arrangement of doubledeep rack, in which the $\mathrm{I} / \mathrm{O}$ point is located in the lower left corner, and there are 16 rows and 12 goods allocation positions each row. Thus, the total number of goods allocation position is $(\mathrm{N})=12 * 16=192$.

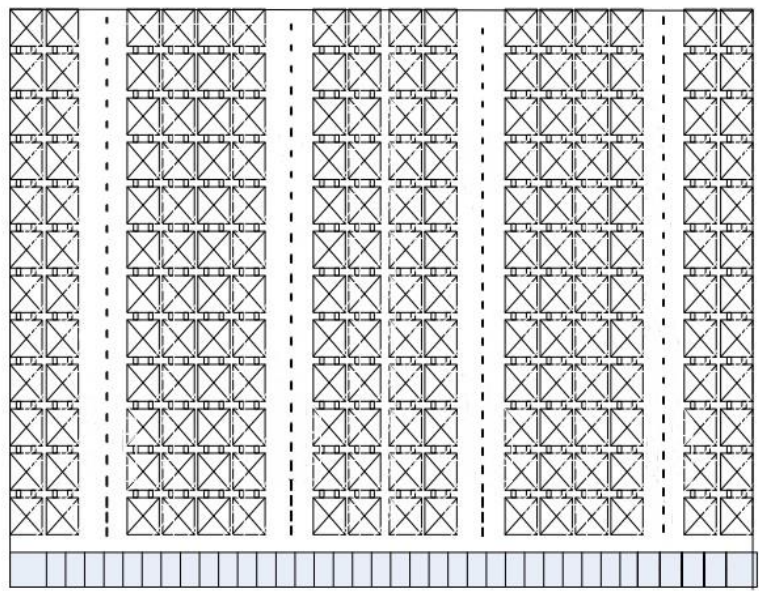

Fig. 1. Double-deep rack display mode

\section{A. Formula Calculation}

Since automated crane only travels horizontally and vertically, the distance of automated crane from $\mathrm{i}$ to $\mathrm{m}$ equals to the sum of horizontal distance and vertical distance:

$$
D_{i, m}=\left|x_{i}-x_{m}\right|+\left|y_{i}-y_{m}\right|
$$

Hence, if automated crane executes a dual command, it will travel from I/O point to the position of goods allocation for storing, then to the position of goods allocation for searching, and then return to I/O point. Suppose the time taken to store cargo from automated crane in the first goods allocation position is equal to that taken to take cargo from the first goods allocation position and put it in automated crane, which is denoted by $\mathrm{t}$, and the time taken to store or retrieve cargo into or from the second goods allocation position is twice over that taken to store or retrieve cargo into or from the first goods allocation position, which is denoted by $2 \mathrm{t}$.

Automated crane can travel in aisle only, and executes dual command. If a warehouse is denoted by a matrix, then aisle occupies the first column, the column coordinates of the first goods allocation $\mathrm{n}$ and of the second goods allocation position are different, and it needs to convert the coordinates of the first and second goods allocation positions into coordinates of aisle for calculating distance (namely changing the horizontal ordinates while maintaining the vertical ordinates unchanged). Suppose $G^{\prime}{ }_{i, m}(R, C)$ refers to the coordinate position of the goods allocation position into or from which storing or retrieving is to be done, and $G_{i, m}(R, C)$ refers to the coordinate position of automated crane in aisle, automated crane travels in land on both sides of which there are racks, and aisle occupies a column, then:

$$
G_{i, m}(R, C)= \begin{cases}G_{i, m}^{\prime}\left(R^{\prime}+2, C\right) & R^{\prime}=1,6,11,16 \\ G_{i, m}^{\prime}\left(R^{\prime}+1, C\right) & R^{\prime}=2,7,12,17 \\ G_{i, m}^{\prime}\left(R^{\prime}-1, C\right) & R^{\prime}=4,9,14,19 \\ G_{i, m}^{\prime}\left(R^{\prime}-2, C\right) & R^{\prime}=5,10,15,20\end{cases}
$$

Case 1: Storage and retrieval goods both are located in the first position, and the second position can be either empty or occupied. Then, the formula is:

$$
T_{i m}^{S R}=2 \times\left(\operatorname{Max}\left\{x_{G i}, x_{G m}\right\}+\operatorname{Max}\left\{y_{i}, y_{m}\right\}\right)+2 t
$$

Case 2: Storage or retrieval goods are located in the second position

i. If storage goods $i$ are located in the second position, and the first position of $\mathrm{i}$ is occupied, then retrieval goods $\mathrm{m}$ are located in the first position. Or storage goods $i$ are located in the first position, retrieval goods $\mathrm{m}$ are located in the second position, and the first position of $\mathrm{m}$ is occupied, then the formula is:

$$
T_{i m}^{S R}=2 \times\left(\operatorname{Max}\left\{x_{G i}, x_{G m}\right\}+\operatorname{Max}\left\{y_{i}, y_{m}\right\}\right)+5 t
$$

ii. If storage goods i are located in the second position, and the first position of $\mathrm{i}$ is empty, then retrieval goods $\mathrm{m}$ are located in the first position. Or storage goods $i$ are located in the first position, retrieval goods $\mathrm{m}$ are located in the second position, and the first position of $\mathrm{m}$ is empty, then the formula is:

$$
T_{i m}^{S R}=2 \times\left(\operatorname{Max}\left\{x_{G i}, x_{G m}\right\}+\operatorname{Max}\left\{y_{i}, y_{m}\right\}\right)+3 t
$$

Case 3: Storage and retrieval goods are located in the second position

i. If storage and retrieval goods are located in the second position, and the first positions of $\mathrm{i}$ and $\mathrm{m}$ both are occupied, then the formula is:

$$
T_{i m}^{S R}=2 \times\left(\operatorname{Max}\left\{x_{G i}, x_{G m}\right\}+\operatorname{Max}\left\{y_{i}, y_{m}\right\}\right)+8 t
$$

ii. If storage and retrieval goods are located in the second position, and the first position of $i$ is occupied and that of $m$ is empty; or the first position of $\mathrm{i}$ is empty, and that of $\mathrm{m}$ is occupied, then the formula is:

$$
T_{i m}^{S R}=2 \times\left(\operatorname{Max}\left\{x_{G i}, x_{G m}\right\}+\operatorname{Max}\left\{y_{i}, y_{m}\right\}\right)+6 t
$$

iii. If storage and retrieval goods are located in the second position, the first positions of $i$ and $m$ both are empty, then the formula is:

$$
T_{i m}^{S R}=2 \times\left(\operatorname{Max}\left\{x_{G i}, x_{G_{m}}\right\}+\operatorname{Max}\left\{y_{i}, y_{m}\right\}\right)+4 t
$$

Case 4: Storage and retrieval goods are in the same side of the aisle.

If storage goods are located in the first position, and retrieval goods in the second position; or storage goods are located in the second position, and retrieval goods in the first position, then the formula is: 


$$
T_{i m}^{S R}=2 \times\left(\operatorname{Max}\left\{x_{G i}, x_{G m}\right\}+\operatorname{Max}\left\{y_{i}, y_{m}\right\}\right)+3 t
$$

For purpose of this paper, emphasis is put on the case where the time taken for storing and that for retrieving are equal, to shorten interaction time by executing dual command and then to shorten the total time.

\section{OPTIMIZATION OF ALGORITHM DESIGN}

This paper focuses on the number of storage goods is equal to the number of retrieval goods, to shorten interaction time by executing dual command and then to shorten the total time.

\section{A. Establish the mathematical model}

Optimization principles:

- Sort goods by turnover rate in an ascending order first (goods of high turnover rate are stored near I/O point);

- Store the same goods in the same row of racks;

- Use the first position of each rack first;

- Store related goods adjacent to each other;

- Place goods of large mass at the bottom of rack to ensure the stability of rack.

A dual command time model was built based on Formula (2). With the model, it can be realized to arrange the position for storing based on determination of the position for retrieving by automated crane's executing a dual command. In this way, the interaction time is shortened, and the total travelling time of automated crane is also shortened. The optimization model is as follows:

Suppose the time taken by stacking crane to travel from $\mathrm{I} / \mathrm{O}$ point to each position is known.

Model parameters: $\mathrm{K}$ refers to the total quantity of goods allocation positions; $\mathrm{N}$ refers to the total amount of goods; tik refers to the time taken to store $\mathrm{i}$ goods to $\mathrm{k}$ position.

Decision variable:

$$
x_{i k}=\left\{\begin{array}{l}
1, \text { goodsi are stored in } \mathrm{k} \text { position } \\
0, \text { otherwise }
\end{array}\right.
$$

A time model of integer programming is formulated as:

$$
\min T^{\prime}=\sum_{i=1}^{N} \sum_{k=1}^{K} t_{i k} x_{i k}
$$

subject to:

$$
\begin{gathered}
\sum_{k=1}^{K} x_{i k}=1 \quad i=1,2 \ldots N \\
x_{i k} \in\{0,1\}
\end{gathered}
$$

Formula (18) means that only one position is allocated to each unit of goods; In Formula (19), xik is a variable of which the value is 0 or 1 .

Suppose during the period t, 10 goods are warehoused, the occupancy rate of warehouse reaches above $85 \%$, and the conditions of positions are as shown in Fig. 2: I/O point is in the lower left corner, grey means being occupied $(\mathrm{O})$, white means being empty $(S)$, and red means retrieval goods (R).

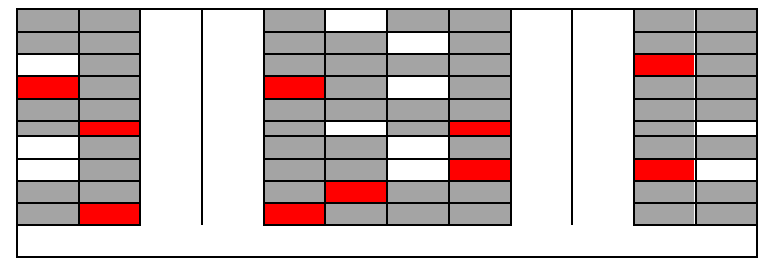

Fig. 2. Position condition of warehouse during the period $t$

Consider the rectangular warehouse in certain coordinates, of which the horizontal axis is in horizontal direction, the vertical axis is in vertical direction, the original point is $\mathrm{I} / \mathrm{O}$ point. Thus, the occupancy of the warehouse indicated with matrix is as below:

$$
\left[\begin{array}{lccc}
t_{1,1} X_{1,1} & t_{1,2} X_{1,2} & \ldots & t_{1,12} X_{1,12} \\
t_{2,1} X_{2,1} & t_{2,2} X_{2,2} & \ldots & t_{2,12} X_{2,12} \\
\cdots & \ldots & \ldots & \ldots \\
t_{10,1} X_{10,1} & t_{10,2} X_{10,2} & \ldots & t_{10,12} X_{10,12}
\end{array}\right]
$$

Suppose $t_{i k}=1$, the occupancy of the warehouse is indicated with a $12 * 10$ matrix, " 0 " means being occupied, and " 1 " means being empty, then

$$
A=\left[\begin{array}{ccccc}
0 & 0 & \ldots & 0 & 0 \\
0 & 0 & \ldots & 0 & 0 \\
1 & 0 & \ldots & 0 & 1 \\
\ldots & \ldots & \ldots & \ldots & \ldots \\
0 & 0 & \ldots & 0 & 0
\end{array}\right]
$$

The empty positions are: $\mathrm{X}_{3,1}, \mathrm{X}_{3,7}, \mathrm{X}_{3,12}, \mathrm{X}_{4,1}, \mathrm{X}_{4,7}, \mathrm{X}_{5,6}$, $\mathrm{X}_{5,12}, \mathrm{X}_{7,7}, \mathrm{X}_{8,1}, \mathrm{X}_{9,7}, \mathrm{X}_{10,6}$.

Step 1: Determine the sequence of retrieval goods, and order the goods from the near to the distant beginning from I/O point:

$\mathrm{X}_{1,2}-\mathrm{X}_{1,5}-\mathrm{X}_{2,6}-\mathrm{X}_{5,2}-\mathrm{X}_{7,1}-\mathrm{X}_{7,5}-\mathrm{X}_{3,8}-\mathrm{X}_{3,11}-\mathrm{X}_{5,8}-\mathrm{X}_{8,11}$.

Step 2: Determine the storage position of goods

Calculate the Manhattan distance between the two points, and work out the shortest route from the determined empty positions of retrieval goods:

$$
D_{12}=\sum_{k=1}^{n}\left|x_{k 1}-x_{k 2}\right|
$$

The goods searched first are $\mathrm{X}_{1,2}: \mathrm{I} / \mathrm{O} \rightarrow \mathrm{X}_{3,1} \rightarrow \mathrm{X}_{1,2} \rightarrow \mathrm{I} / \mathrm{O}$.

Step 3: Calculate the time taken to execute a dual command. 
Step 4: Keep updating the positions in warehouse after Steps 1, 2, 3 are finished until the commands of storing and retrieving are completed, and calculate the total time.

\section{B. Simulation Experiment (after optimization \& storage designation at random)}

Automated crane, at the time of executing dual command, should follow the principle of retrieving after storing. That's to say, storing operation should go before retrieval operation. The optimization method is to select a position for storing to which the time to reach is shortest, for which the distance to both I/O point and the position of designated retrieval goods should be considered. Thus, as is shown in Fig.3, the optimal position is in the middle of the two points. Horizontal coordinate refers to the times of automated crane executing dual command, and vertical coordinate refers to the expected time taken by automated crane to finish the operations of storing and retrieving.

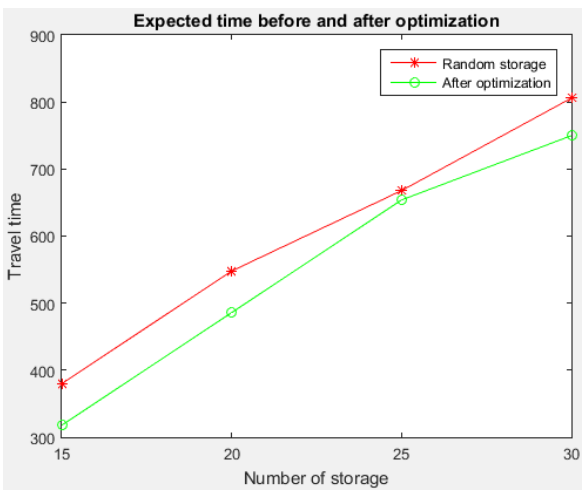

Fig. 3. Simulation results graph

\section{CONCLUSION}

This paper mainly aims to solve the problem of dynamic goods allocation position distribution in automated warehousing system, and realize rational utilization of resources. Firstly, to save total warehousing cost, double-deep racks are applied to double the position density, having realized compact warehousing and enhanced the compactness of warehouse space; secondly, the method of designating automated crane to execute dual command is adopted, having shortened interaction time, raised operation efficiency, and saved space cost and time cost.

\section{REFERENCES}

[1] Iris F A, Kees Jan Roodbergen. Layout and Control Policies for Cross Docking Operation [J]. Computers \& Industrial Engineering, 2011, 61(4): 911-919.

[2] Yang Haigen, Zhan Zhihuan, Fu Xiao and Wang Yong. Control System Design for Electric-Intensive Mobile Rack [J]. International Journal of Control and Automation, 2015, (7): 99-112.

[3] HwanSeong Kim, EunJi Kim, KyoungMi Cheon. Optional Heuristics Algorithm of Single Job-order for Mobile Racks [J]. MCM, 2015, 257.

[4] Warren H. Hausman, Leroy B. Schwarz, Stephen C. Graves. Optimal Storage Assignment in Automatic Warehousing Systems[J]. Management Science, 1976.

[5] Seval Ene. Nursel Ozturk. Storage Location Assignment and Order Picking Optimization in the Automotive Industry[J] . Int J Adv Manuf Technol, 2012, 60: 787-797.

[6] Russell D. Meller, Anan Mungwattana. Multi-Shuttle Automated Storage/Retrieval Systems[J]. IIE Transactions, 1997,29:925-938.

[7] Deng Aimin, Cai Jia, Mao Lang. Research of Goods Allocation Position Optimization of Automated Stereoscopic Warehouse from the Perspective of Time [J]. Chinese Journal of Management Science, 2013, 21 (6): 107-112

[8] Gu J, Goetschalckx M, McGinnis L F. Research on Warehouse Operation: A Comprehensive Review [J]. Europe Journal of Operation Research, 2007, 177(1): 1-21.

[9] Kees Jan Roodbergena,*, Iris F. A. Visb,1. A Survey of Literature an Automated Storage and Retrieval Systems [J]. European Journal of Operational Research, 2009, 194: 343 362.

[10] Shen Guwen. Research of Performance and Operation Strategy of Compact Warehousing System [D]. Huazhong University of Science and Technology: Management Science and Engineering, Doctoral dissertation 2014 